\title{
Expression of human 8-oxoguanine DNA glycosylase (hOGG1) in follicular lymphoma
}

\author{
Andrea M Sheehan ${ }^{1, *}$, David K McGregor ${ }^{1, *}$, Ankita Patel ${ }^{2}$, Vinod Shidham³, \\ Chung-Yang Fan ${ }^{4}$ and Chung-Che Chang ${ }^{5}$
}

\begin{abstract}
${ }^{1}$ Department of Pathology, Baylor College of Medicine, Houston, TX, USA; ${ }^{2}$ Department of Molecular and Human Genetics, Baylor College of Medicine, Houston, TX, USA; ${ }^{3}$ Department of Pathology, Medical College of Wisconsin, Milwaukee, WI, USA; ${ }^{4}$ Department of Pathology, University of Arkansas, Little Rock, AR, USA and ${ }^{5}$ Department of Pathology, The Methodist Hospital, Weil Medical College, Cornell University, Houston, TX, USA
\end{abstract}

\begin{abstract}
The human homologue of the yeast DNA repair enzyme 8-oxoguanine DNA glycosylase (hOGG1) repairs oxidatively damaged guanosine nucleotides in DNA. This enzyme is highly expressed in reactive germinal centers, where lymphoid cells are under oxidative stress, and has been thought to protect lymphocytes from mutation. As a first step to investigate the role of hOGG1 in lymphomagenesis, we evaluated hOGG1 expression in follicular lymphoma. Immunohistochemistry was performed on formalin-fixed paraffin-embedded tissue of 28 follicular lymphoma cases (16 grade 1, seven grade 2, and five grade 3) to evaluate the expression of hOGG1 in neoplastic follicles. Reactive germinal centers of non-neoplastic tonsil and lymph node tissue were also examined. Fluorescent-in-situ hybridization (FISH) was performed using a DNA probe from BAC clone RP11266J6 corresponding to 3p25, where the hOGG1 gene resides, to evaluate for the presence or absence of a deletion. In reactive germinal centers, the majority of centroblasts and centrocytes were positive for hOGG1. In contrast, the majority (21 of 28 or $75 \%$ ) of follicular lymphoma cases showed absent/minimal expression of hOGG1. Only four of 28 (14\%) follicular lymphoma cases revealed the same levels of hOGG1 expression as reactive germinal centers. There was no correlation between hOGG1 expression and histologic grade. None of the 16 cases evaluated by FISH showed a deletion of hOGG1. Furthermore, absent/minimal hOGG1 expression was observed in four of six Bcl-2-negative follicular lymphoma cases. Our findings suggest that absent/minimal hOGG1 expression occurs in the majority of follicular lymphomas. The downregulation of hOGG1 does not appear to be due to a deletion of the hOGG1 locus. Additionally, finding absent/minimal hOGG1 expression in a subset of Bcl-2-negative follicular lymphomas suggests that hOGG1 may have utility in diagnosing Bcl-2negative follicular lymphomas.

Modern Pathology (2005) 18, 1512-1518. doi:10.1038/modpathol.3800461; published online 29 July 2005
\end{abstract}

Keywords: follicular lymphoma; hOGG1; immunohistochemistry

The human homologue of the yeast DNA repair enzyme, 8-oxoguanine-DNA glycosylase (hOGG1), excises 8-oxoguanine, an oxidative form of the guanine nucleotide. ${ }^{1}$ HOGG1 shares a $38 \%$ identity with the yeast OGG1. It has been localized to chromosome 3p25 by fluorescent-in-situ hybridization (FISH) by Radicella et al in $1997,{ }^{2}$ and it was cloned by Rosenquist et $a l^{3}$ in 1997. This DNA

Correspondence: Dr C-C Chang, MD, PhD, Department of Hematopathology, The Methodist Hospital, Weil Medical College, Cornell University, 6565 Fannin Street, MS 205, Houston, TX 77030, USA.

E-mail: jeffchang@tmh.tmc.edu

*Both these authors contributed equally to this work.

Received 3 February 2005; revised 9 June 2005; accepted 10 June 2005; published online 29 July 2005 glycosylase/apurinic lyase repairs oxidative DNA damage, specifically 8-oxoguanine/C base pairs. 8-hydroxyguanine is highly mutagenic via GC to TA transversions ${ }^{4}$ and is generated by oxidative stresses as well as normal cellular metabolism. ${ }^{5}$

It is believed that hOGG1's role of excising 8-oxoguanine protects DNA against the mutagenic effects of oxidative damage and decreased cell survival from radiation-induced damage. ${ }^{6}$ Supporting hOGG1's role of hindering mutagenesis is that certain a genetic polymorphism of hOGG1, Ser326Cys, has been implicated in an increased incidence of adenocarcinoma of the $\operatorname{lung}^{7}$ and squamous cell carcinoma of the lung ${ }^{8,9}$ in patients with the Cys/Cys genotype, as compared to Ser/Cys or Ser/Ser genotypes. Studies on oxidative damage and genetic polymorphisms of hOGG1 have shown 
that hOGG1 may play a role in solid tumor carcinogenesis including breast cancer, ${ }^{10}$ pancreatic adenocarcinoma, ${ }^{11}$ and squamous cell carcinoma of the head and neck. ${ }^{12}$ One recent review by Weiss et $a l^{13}$ indicates that there is epidemiologic evidence that hOGG1 genotype may be related to risk of esophageal, head and neck, lung, nasopharyngeal, orolaryngeal, and prostate cancer. Oxidative damage and hOGG1 expression has also been studied in non-neoplastic diseases. Iida et $a l^{14}$ have recently reported that decreased hOGG1 expression associated with neurofibrillary tangles is observed in patients with Alzheimer's dementia suggesting a role for oxidative damage in the development of this disease.

A study by Kuo and Sklar demonstrated that hOGG1 is highly expressed within germinal center B cells at levels greatly exceeding that found in other tissues, and that expression of this gene is most abundant within the dark, centroblast-rich zone of germinal centers. ${ }^{15}$ The expression of hOGG1 in the dark zone of germinal centers may serve as a protective mechanism for the germinal center lymphocytes from oxidative damage-induced mutations arising as a result of processes such as antigen stimulation or release of oxygen-free radicals by macrophages in the germinal centers. To our knowledge, expression of $h O G G 1$ in lymphomas has not been studied.

The aim of this study was two-fold. The first aim was to evaluate the pattern of hOGG1 expression in different grades of follicular lymphoma, a lymphoma of germinal center B-cell origin, and so doing, preliminarily evaluate the role of hOGG1 in lymphomagenesis. The second aim was to use FISH to evaluate for the presence or absence of a deletion of the region containing hOGG1 (3p25) and so doing, examine whether the mechanism for loss of hOGG1 expression may be a deletion vs modulation of gene expression by some other mechanism.

\section{Materials and methods}

\section{Patients}

In all, 28 cases (13 males, 15 females; age range: $37-$ 90 years old, mean 64 years old) of follicular lymphoma were retrieved from the files of the Department of Pathology, Medical College of Wisconsin, Milwaukee, WI, USA. The morphologic diagnosis of follicular lymphoma was reviewed and confirmed. Then, the cases were classified into grades 1, 2, and 3 according to criteria put forth by the WHO. ${ }^{16}$ The cohort of cases included 16 grade 1, seven grade 2 , and five grade 3 follicular lymphomas. As a control non-neoplastic lymph nodes and tonsils were examined. The Medical College of Wisconsin Institutional Review Board approved the study protocol.

\section{Immunohistochemistry}

Formalin-fixed paraffin-embedded tissue was available in all of the cases. Paraffin sections were mounted on Fisherbrand/Plus Superfrost Precleaned slides, heated at $60^{\circ} \mathrm{C}$ for $30 \mathrm{~min}$, deparaffinized and rehydrated. Tissue sections were incubated with the rabbit polyclonal antibody to hOGG1 (Novus Biologicals, Littleton, CO, USA) and Bcl-2 (clone: 124, Dako, Carpinteria, CA, USA) using the antigen retrieval techniques as described below. Slides were incubated for $30 \mathrm{~min}$ in 50:50 methanol peroxide then the antigen was retrieved in citrate buffer, pH 8.0 (hOGG1) or EDTA buffer, pH 10.0 (Bcl-2) for $30 \mathrm{~min}$ at $98^{\circ} \mathrm{C}$ then cooled for $20 \mathrm{~min}$. Nonspecific protein-binding sites were blocked by serum for $5 \mathrm{~min}$ and the primary antibody added at different dilutions (1:50 for hOGG1 and 1:100 for Bcl-2). All slides were stained using the Dako automated immunostainer (Dako, Carpinteria, CA, USA). The slides were incubated for $15 \mathrm{~min}$ in labeled streptavidin-biotin (LSAB) for linking and labeled subsequently with diaminobenzidine chromogen. Appropriate positive and negative controls were used in all cases.

\section{Interpretation of Immunohistochemistry Results}

As a control, reactive germinal centers of nonneoplastic tonsils and lymph nodes $(n=10)$ were examined to assess the percentage of centroblasts and centrocytes positive for hOGG1. The percentage of either centroblasts or centrocytes positive for hOGG1 was determined in a total of 1487 cells in 15 germinal centers. Morphologic criteria published by the WHO were used for identification of centroblasts and centrocytes. ${ }^{16}$

Expression of Bcl-2 and hOGG1 was systematically reviewed by two of the authors (DM and CC) independently. The percentage of cells exhibiting positive staining for hOGG1 and Bcl-2 was evaluated by examining 10 high-power fields $(\times 400)$ in the follicular areas. The percentage of cells staining for hOGG1 (nuclear staining) or Bcl-2 (cytoplasmic staining) in lymphoma cases were graded as follows: $0-5 \%=0,6-20 \%=1+$, $21-50 \%=2+,>50 \%=3+$. Follicular lymphomas showing positive staining in $\leq 5 \%$ of tumor cells were considered as having absent/minimal expression. The CD3 stain (highlighting the $\mathrm{T}$ cells) performed as part of the initial diagnostic workup in these cases was reviewed in conjunction with bcl2 stain. The number of bcl-2-positive lymphoma cells was estimated by subtracting total CD3 $+\mathrm{T}$ cells from bcl-2-positive cells. Additionally, the bcl-2-positive lymphoma cells had lower staining intensity than bcl-2-positive $\mathrm{T}$ cells in most cases. This difference in staining intensity also allowed an accurate estimate of the percentage of bcl-2-positive lymphoma cells. 
This grading system led to agreement between the two observers for over $95 \%$ of staining results. In the case of discrepant results, the two investigators reevaluated the slide and eventually reached a consensus. The Bcl-2-positive $\mathrm{T}$ cells were used as a positive internal control to confirm that the antigens were retrievable for all the cases. Since hOGG1 is only expressed in normal germinal center B cells, there was no positive internal control for hOGG1 staining in the follicular lymphoma cases with absent hOGG1 expression. However, appropriate controls were performed and evaluated with each staining run to ensure that the antibody was working correctly.

\section{FISH Study for Determining the Copy Number of the hOGG1 Gene}

\section{Preparation of probes}

A search of the NCBI human genome project website identified the localization of hOGG1 to chromosome 3p25 and BAC clone RP11-266J6. The clone was inoculated into $15 \mathrm{ml}$ of $\mathrm{LB}$ broth medium (QBiogene, Carlsbad, CA, USA) containing $12.5 \mu \mathrm{g} / \mathrm{ml}$ of choramphenicol and grown overnight at $37^{\circ} \mathrm{C}$ with shaking at $200 \mathrm{rpm}$. BAC DNA was extracted using the standard alkaline lysis protocol.

\section{Labeling and reprecipitation of DNA probes}

In all, $1 \mu \mathrm{g}$ of BAC DNA was labeled with biotin-14dUTP (Roche diagnostics, Indianapolis, IN, USA), using the standard Nick translation protocol. The labeled probe was resuspended in $10 \mu \mathrm{l}$ of hybridization solution $(50 \%$ formamide, $10 \%$ dextran sulfate, 2XSSC, $\mathrm{dH}_{2} \mathrm{O}$ ) and stored at $-20^{\circ} \mathrm{C}$ until needed.

\section{FISH}

The probe was tested on known normal controls, using previously karyotyped peripheral blood lymphocytes, and metaphases were analyzed to verify hybridization to the appropriate chromosomal location. The FISH study was performed in 16 of 28 follicular lymphoma cases. Normal non-neoplastic tonsil was used as a negative control. Tissue sections ( $5 \mu \mathrm{m}$ )were obtained on plus slides. Deparaffinization was accomplished using Citrusolv (Fisher Scientific, Pittsburgh, PA, USA) at room temperature for 10-15 min followed by air drying. The sections were dehydrated at room temperature in 75,85 , and $95 \%$ ethanol for 2 min each and were then air dried. The sections were treated with $2 \times$ TissueDigest Solution (Insitus biotechnologies, Albuquerque, $\mathrm{NM}, \mathrm{USA}$ ) in a $37^{\circ} \mathrm{C}$ water bath for $2 \mathrm{~h}$. Slides were dehydrated in an ethanol series again as mentioned previously. In all, $20 \mu \mathrm{l}$ of a $50 \%$ glycerol $/ 1 \times$ SSC solution was placed over the tissue area and covered with a glass cover slip. The slides were baked in a $90^{\circ} \mathrm{C}$ oven for $5 \mathrm{~min}$ and allowed to cool. Slides were washed in $2 \times \mathrm{SSC}$ at room temperature for $2 \mathrm{~min}$ followed by dehydration in 75,85 , and $95 \%$ ethanol at room temperature. After air drying, $5 \mu \mathrm{l}$ of probe was applied to the tissue area, the slides were cover slipped and secured with rubber cement. The slides were placed in a humidified chamber (HYBrite, Vysis, Inc., Downers Grove, IL, USA), denatured at $80^{\circ} \mathrm{C}$ for $10 \mathrm{~min}$, and hybridized overnight at $37^{\circ} \mathrm{C}$.

After overnight hybridization, the slides were washed in $0.4 \times \mathrm{SSC} / 0.3 \% \mathrm{NP}-40$ for $2 \mathrm{~min}$ at $72^{\circ} \mathrm{C}$ and then washed in $2 \times \mathrm{SSC} / 0.1 \% \mathrm{NP}-40$ for $2 \mathrm{~min}$ at room temperature. Detection and amplification of the biotin signals were accomplished with incubation of the slides with FITC-avidin (Vector laboratories, Inc., Burlingame, CA, USA). The slides were counterstained with $15 \mu \mathrm{l}$ of DAPI (Vector laboratories, Inc., Burlingame, CA, USA), cover slipped, and analyzed using a Zeiss Axiophot fluorescent microscope equipped with both single- and tripleband pass filters. Digital images were captured by a Power Macintosh G3 system and MacProbe version 4.0 (Applied Imaging, San Jose, CA, USA).

\section{Scoring of signals}

The slides were evaluated for the presence of signals. In all, 200 cells were scored for each case, looking for the presence of one, two, or more signals. A total of 400 cells were scored for normal nonneoplastic tonsillar tissue, and eight $(2 \%)$ were found to have only one signal. Cases with more than $5 \%$ of cells exhibiting no or one signal were considered positive for a deletion, to increase specificity. Cases were photographed.

\section{Results}

In reactive tonsils and lymph nodes $(n=10)$, mantle zone B cells and interfollicular lymphocytes showed minimal hOGG1 expression ( $\leq 5 \%$ ), while germinal centers revealed high-level expression with $85 \%$ of centroblasts and $63 \%$ of centrocytes positive for hOGG1 (Table 1 and Figure 1a and b). In contrast, the majority of follicular lymphoma cases $(22 / 28$ or $79 \%$ vs $0 / 10$ of reactive lymph nodes or tonsils, $P<0.0001$, Fisher's exact) showed absent or minimal $(0-5 \%)$ staining of $h O G G 1$ in the neoplastic follicles (Table 2). These 22 cases with minimal or absent hOGG1 expression included 12/16 grade 1, 6/7 grade 2 , and $4 / 5$ grade 3 follicular lymphomas. Two (both grade 1) of 28 cases $(7 \%)$ showed low to moderate level hOGG1 expression $(1+$ to $2+)$. Four

Table 1 Expression of hOGG1 in normal tonsil and lymph node tissue

\begin{tabular}{lcc}
\hline & Centroblasts & Centrocytes \\
\hline Tonsils & $90 \%(340 / 378)$ & $65 \%(338 / 519)$ \\
Lymph nodes & $72 \%(125 / 172)$ & $60 \%(252 / 418)$ \\
Overall & $85 \%(465 / 550)$ & $63 \%(590 / 937)$
\end{tabular}



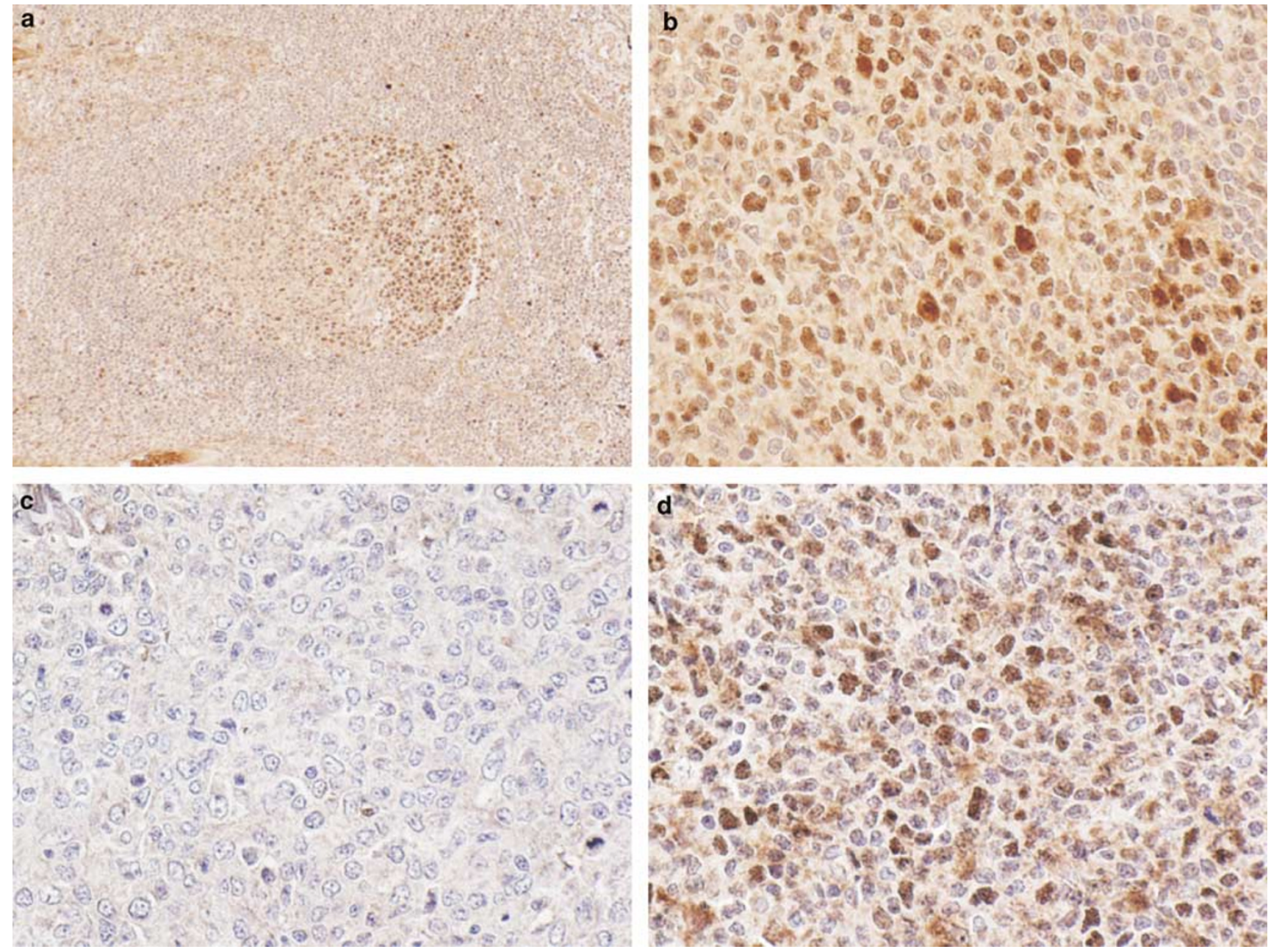

Figure 1 Expression of hOGG1 in reactive germinal centers and examples of hOGG1 expression in follicular lymphoma cases. (a and b) hOGG1 expression in a reactive germinal center with accentuated staining of the dark zone $(\mathbf{a}, \times 200)$ and with positive staining present in the nuclei of both centroblasts and centrocytes $(\mathbf{b}, \times 400)$; (c) negative staining for hOGG1 by immunohistochemistry (IHC) in a grade 3 follicular lymphoma $(\times 400)$; and (d) positive staining for hOGG1 by IHC in a grade 1 follicular lymphoma $(\times 400)$.

(two grade 1, one grade 2 , and one grade 3 ) of 28 cases $(14 \%)$ showed a high level of hOGG1 expression $(3+)$ comparable to reactive germinal centers. In general, cases with hOGG1 expression showed strong intensity of staining. Examples of hOGG1 expression in lymphoma cases are shown in Figure 1c and $d$. There was no correlation between the hOGG1 expression status and the histologic grade of follicular lymphoma.

Most of the follicular lymphoma cases for which Bcl-2 data were available were bcl-2 positive as follows: $12 / 15(80 \%)$ grade $1,5 / 7(71 \%)$ grade $2,3 / 4$ $(75 \%)$ grade 3 . Six of 26 cases $(23 \%)$ showed absent staining of Bcl-2 (Table 2). These included three grade 1 , two grade 2 , and one grade 3 follicular lymphomas. Of those six cases, four $(67 \%$, two grade 1 , one grade 2 and one grade 3 ) showed absent/ minimal expression of hOGG1. One case (grade 1) showed a moderate level $(2+)$ of hOGG1 expression, and the remaining one (grade 2) showed a strong level $(3+)$ of hOGG1 expression. No correla- tion between hOGG1 expression and Bcl-2 expression was observed.

FISH for the hOGG1 probe showed a normal dual green signal pattern in 196 of 200 (98\%) cells scored in normal tonsillar tissue indicating that gene copy number could be reliably determined by FISH with tissue sections. Of 16 follicular lymphoma cases tested for $h O G G 1$, four $(25 \%$, two grade 1 , one grade 2 , and one grade 3) showed positive hOGG1 expression, and $12(75 \%$, four grade 1 , four grade 2 , and four grade 3) showed absent or minimal hOGG1 expression. All cases showed a normal signal pattern (ie two copies of the hOGG1 gene in each cell) in $97-100 \%$ of cells scored, indicating that no deletion was present (Table 2 and Figure 2).

\section{Discussion}

Our results indicate that downregulation of hOGG1 expression at the protein level is a common feature 
Table 2 Results of expression of Bcl-2 and hOGG1 by immunohistochemistry and fluorescent in situ hybridization (FISH)

\begin{tabular}{|c|c|c|c|c|c|}
\hline Case & Grade & $\begin{array}{c}\text { hOGG1 } \\
\text { immunohistochemistry }\end{array}$ & $B c l-2$ & $\begin{array}{l}\text { hOGG1 FISH percent dual signal } \\
\text { pattern (200 cells scored) }\end{array}$ & $\begin{array}{l}\text { hOGG1 FISH } \\
\text { interpretation }\end{array}$ \\
\hline 1 & 1 & $1 t^{\mathrm{a}}$ & $3+$ & 100 & No deletion \\
\hline 2 & 1 & 0 & $3+$ & 97 & No deletion \\
\hline 3 & 1 & 0 & $3+$ & $\mathrm{ND}^{\mathrm{b}}$ & ND \\
\hline 4 & 1 & 0 & ND & 100 & No deletion \\
\hline 5 & 1 & $2+$ & 0 & 98 & No deletion \\
\hline 6 & 1 & 0 & $3+$ & 100 & No deletion \\
\hline 7 & 1 & 0 & $2+$ & 100 & No deletion \\
\hline 8 & 1 & $3+$ & $3+$ & ND & ND \\
\hline 9 & 1 & 0 & $2+$ & ND & ND \\
\hline 10 & 1 & 0 & 0 & ND & ND \\
\hline 11 & 1 & 0 & 0 & ND & ND \\
\hline 12 & 1 & 0 & $3+$ & ND & ND \\
\hline 13 & 1 & 0 & $3+$ & ND & ND \\
\hline 14 & 1 & $3+$ & $3+$ & ND & ND \\
\hline 15 & 1 & 0 & $3+$ & ND & ND \\
\hline 16 & 1 & 0 & $2+$ & ND & ND \\
\hline 17 & 2 & $3+$ & 0 & 100 & No deletion \\
\hline 18 & 2 & 0 & $3+$ & ND & ND \\
\hline 19 & 2 & 0 & $1+$ & 99 & No deletion \\
\hline 20 & 2 & 0 & 0 & 100 & No deletion \\
\hline 21 & 2 & 0 & $3+$ & 100 & No deletion \\
\hline 22 & 2 & 0 & $3+$ & 100 & No deletion \\
\hline 23 & 2 & 0 & $3+$ & ND & ND \\
\hline 24 & 3 & 0 & $2+$ & 100 & No deletion \\
\hline 25 & 3 & $3+$ & ND & 100 & No deletion \\
\hline 26 & 3 & 0 & $3+$ & 98 & No deletion \\
\hline 27 & 3 & 0 & 0 & 99 & No deletion \\
\hline 28 & 3 & 0 & $3+$ & 99 & No deletion \\
\hline
\end{tabular}

${ }^{\mathrm{a}}$ Scoring system for hOGG1 and Bcl-2 immunohistochemistry: $\leq 5 \%=0$ (negative), $6-20 \%=1+, 21-50 \%=2+,>50 \%=3+$. $\mathrm{b}_{\mathrm{ND}}$ not done.

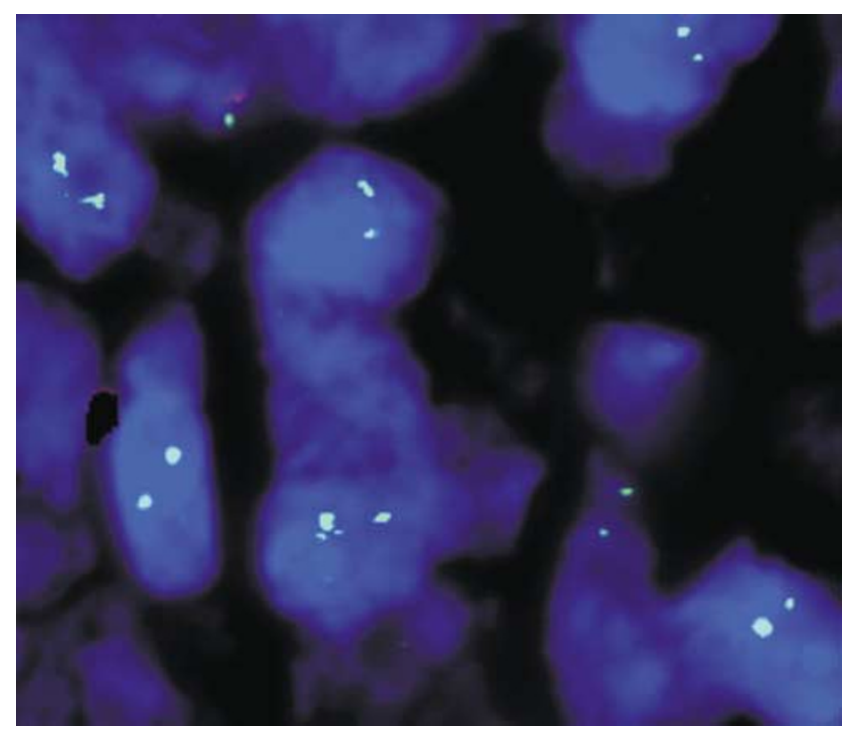

Figure 2 An example of FISH for hOGG1 in follicular lymphoma cases $(\times 1000)$. In this case, two green signals are present and visible in $>97 \%$ of cells indicating no deletion of the 3 p25 locus.

for all histologic grades of follicular lymphoma. In this study, normal reactive germinal centers showed strong expression of hOGG1 with increased expression of hOGG1 in centroblasts (85\%) compared to centrocytes $(63 \%)$. This phenomenon is consistent with the preferential expression of hOGG1 in the dark or centroblast-rich zone of germinal centers as previously described by Kuo and Sklar ${ }^{15}$ who performed in situ hybridization analysis of GCN6 (hOGG1) expression with a cDNA probe on formalin-fixed paraffin-embedded sections of human tonsils. This confirms that the immunohistochemistry approach used in this study is reliable in determining the level of hOGG1 expression in paraffin-embedded lymphoid tissues. We found that in contrast to the strong expression of hOGG1 observed in non-neoplastic germinal centers, most follicular lymphomas of all histologic grades showed absent/decreased hOGG1 expression. Only $4 / 28(14 \%)$ cases had a level of hOGG1 expression comparable to that of normal reactive germinal centers. Lymphoid cells in normal germinal centers are under oxidative stress due to antigen stimulation and may require increased expression of hOGG1 to protect them from oxidative damage-induced mutation.

Thus, the downregulation of hOGG1 expression in most cases of follicular lymphoma of all histologic grades suggests that there may be a predisposition to genetic mutations due to the accumulation of oxidatively damaged DNA in these cases. These mutations, particularly when involving important tumor suppressor genes or oncogenes, may subsequently lead to the development of follicular 
lymphoma. It is unlikely, however, that downregulation of $h O G G 1$ by itself leads to the formation of follicular lymphoma since a recent study shows that somatic hypermutation of immunoglobulin genes, a key function of germinal center B cells, remain intact in the absence of hOGG1 gene expression in knockout mice. ${ }^{1}$ Also, these knockout mice do not have an increased rate of developing malignancy. ${ }^{1,17}$ Additionally, although the number of cases is relatively small in this study, hOGG1 expression in follicular lymphoma did not correlate with disease stage at presentation, overall survival, or response to therapy (data not shown). Taken together, our findings suggest that downregulation of hOGG1 expression may be an early event in lymphomagenesis. The exact role of hOGG1 in the development of follicular lymphoma remains to be illustrated by further studies.

The mechanism leading to the downregulation of hOGG1 expression in follicular lymphoma remains unclear. As none of the 16 cases tested by FISH showed a deletion of hOGG1, the mechanism for decreased expression of hOGG1 in follicular lymphoma may be secondary to modulation of gene expression rather than deletion of the genetic locus. Our FISH results agree with previous cytogenetic studies indicating that abnormalities of $3 p$, where hOGG1 resides, are rarely observed in follicular lymphomas, although complex numerical and structural karyotypic abnormalities, in addition to the $\mathrm{t}(14 ; 18)(\mathrm{q} 32 ; \mathrm{q} 21)$, are typical of follicular lymphomas. ${ }^{18,19}$ We, however, cannot exclude the possibility that there may be a microdeletion of the hOGG1 locus accounting for loss of expression of hOGG1. Since a BAC clone was chosen for this study, the size of a deletion detectable by this method is determined by the size of the BAC. BAC clones are typically $150-250 \mathrm{~kb}$, and the length of BAC clone $\mathrm{RP} 11-266 \mathrm{J6}$ used in this study is $185.07 \mathrm{~kb}$. Therefore, a microdeletion smaller than $185 \mathrm{~kb}$ would probably not be detected by this method. Smaller probes can be used; however, the sensitivity and specificity would decrease. Of note, Fan et a $1^{12}$ have reported that majority $(11 / 18$ or $61 \%)$ of cases of head and neck squamous cell cancers show allelic loss of the hOGG1 gene, using PCR-based analysis of two intragenic single nucleotide polymorphism sites, located in intron 4 and exon 7 of the gene. The deletion correlates with decreased hOGG1 expression. However, this approach is only suitable for patients $(40 \%$ or $18 / 45$ in the study by Fan et al) with informative polymorphic loci of hOGG1. Alternatively, epigenetic changes, such as hypermethylation of promoter regions, may result in the decreased hOGG1 expression. For example, hypermethylation of $\mathrm{O}^{6}$-methylguanine-DNA methyltransferase (MGMT), another DNA repair gene, leading to decreased MGMT expression has been shown in head and neck squamous cell cancers, ${ }^{20} \mathrm{~B}$ cell malignancies ${ }^{21}$ and immunodeficiency-related lymphomas. ${ }^{22}$ Indeed, hypermethylation of MGMT in lymphomas has been associated with chemosensitivity to alkylating agents and better survival. ${ }^{23}$ However, preliminary data in a study by CY Fan et $a l^{24}$ found that promoter hypermethylation of the hOGG1 gene detected in 14 of $17(82.3 \%)$ cases of head and neck squamous cell cancers did not correlate with decreased hOGG1 mRNA levels. Further studies are required to illustrate the mechanisms leading to the downregulation of hOGG1 in follicular lymphoma.

In addition to its possible role in the development of follicular lymphoma, hOGG1 expression may have utility in diagnosing follicular lymphomas that are Bcl-2 negative. Bcl-2 has become an important marker to differentiate atypical reactive germinal centers (Bcl-2 negative) from neoplastic follicles in follicular lymphoma (Bcl-2 positive). ${ }^{25}$ However, a small percentage of follicular lymphomas, particularly those that are grade 3, may lack immunohistochemical expression of Bcl-2 resulting in a diagnostic dilemma for pathologists. ${ }^{26}$ The rate of Bcl-2 expression in grade 2 and 3 follicular lymphomas in this study is comparable with what has been reported previously in the literature with a larger number of cases by Lai et al. ${ }^{27}$ However, the rate of bcl-2 expression is lower in grade I follicular lymphomas in the current study than the study by Lai et al. ${ }^{27}$ This is likely due to sampling, as the number of cases in this study is relatively small compared to that by Lai et al. ${ }^{27}$ The same clone and similar antigen-retrieval techniques were used in both studies.

In this study, there were six of 26 cases (23\%) that lacked Bcl-2 expression. Four of these six cases $(67 \%)$ were also negative for hOGG1 expression. Of interest, these four cases included follicular lymphomas of all three grades. These findings suggest that absent/minimal hOGG1 expression may be an important diagnostic marker for Bcl-2-negative follicular lymphomas since normal germinal centers strongly express $h O G G 1$, as shown in the normal controls (tonsils and reactive lymph nodes). Further studies with more cases will be necessary to investigate hOGG1's utility as a diagnostic tool for follicular lymphoma.

In summary, our findings suggest that decreased hOGG1 expression occurs in the vast majority of follicular lymphomas. The downregulation of hOGG1 expression does not appear to be due to a deletion of the hOGG1 locus. Additionally, finding absent/minimal hOGG1 expression in a subset of Bcl-2-negative follicular lymphomas suggests that hOGG1 may have utility in diagnosing Bcl-2negative follicular lymphomas.

\section{Acknowledgements}

This work was previously presented in part at the USCAP annual meeting in 2004 in Vancouver, 
Canada and at the USCAP annual meeting in 2005 in San Antonio, TX, USA.

\section{Duality of interest}

None declared.

\section{References}

1 Winter DB, Phung QH, Zeng X, et al. Normal somatic hypermutation of Ig genes in the absence of 8hydroxyguanine-DNA glycosylase. J Immunol 2003; 170:5558-5562.

2 Radicella JP, Dherin C, Desmaze C, et al. Cloning and characterization of $h O G G 1$, a human homolog of the OGG1 gene of Saccharomyces cerevisiae. Proc Natl Acad Sci USA 1997;94:8010-8015.

3 Rosenquist TA, Zharkov DO, Grollman AP. Cloning and characterization of a mammalian 8-oxoguanine DNA glycosylase. Proc Natl Acad Sci USA 1997; 94:7429-7434.

4 Le Page F, Margot A, Grollman AP, et al. Mutagenicity of a unique 8-oxoguanine in a human Ha-ras sequence in mammalian cells. Carcinogenesis 1995;16:27792784 .

5 Boiteux S, Radicella JP. The human OGG1 gene: structure, functions, and its implication in the process of carcinogenesis. Arch Biochem Biophys 2000;377:1-8.

6 Hyun JW, Cheon GJ, Kim HS, et al. Radiation sensitivity depends on $O G G 1$ activity status in human leukemia cell lines. Free Radic Biol Med 2002;32: 212-220.

7 Ishida T, Takashima R, Fukayama M, et al. New DNA polymorphisms of human MMH/OGG1 gene: prevalence of one polymorphism among lung-adenocarcinoma patients in Japanese. Int J Cancer 1999;80:18-21.

8 Sugimura $\mathrm{H}$, Kohno T, Wakai K, et al. hOGG1 Ser326Cys polymorphism and lung cancer susceptibility. Cancer Epidemiol Biomarkers Prev 1999;8: 669-674.

9 Le Marchand L, Donlon T, Lum-Jones A, et al. Association of the hOGG1 Ser326Cys polymorphism with lung cancer risk. Cancer Epidemiol Biomarkers Prev 2002;11:409-412.

10 Li D, Zhang W, Zhu J, et al. Oxidative DNA damage and 8-hydroxy-2-deoxyguanosine DNA glycosylase/ apurinic lyase in human breast cancer. Mol Carcinog 2001;31:214-223.

11 Li D, Firozi PF, Zhang W, et al. DNA adducts, genetic polymorphisms, and K-ras mutation in human pancreatic cancer. Mutat Res 2002;513:37-48.

12 Fan CY, Liu KL, Huang HY, et al. Frequent allelic imbalance and loss of protein expression of the DNA repair gene hOGG1 in head and neck squamous cell carcinoma. Lab Invest 2001;81:1429-1438.

13 Weiss JM, Goode EL, Ladiges WC, et al. Polymorphic variation in $h O G G 1$ and risk of cancer: a review of the functional and epidemiologic literature. Mol Carcinog 2005;42:127-141.
14 Iida T, Furuta A, Nishioka K, et al. Expression of 8oxoguanine DNA glycosylase is reduced and associated with neurofibrillary tangles in Alzheimer's disease brain. Acta Neuropathol (Berl) 2002;103:20-25.

15 Kuo FC, Sklar J. Augmented expression of a human gene for 8-oxoguanine DNA glycosylase (MutM) in B lymphocytes of the dark zone in lymph node germinal centers. J Exp Med 1997;186:1547-1556.

16 Nathwani BN, Harris NL, Weisenburger $\mathrm{D}$, et al. Follicular lymphoma. In: Jaffe ES, Harris NL, Stein H, Vardiman JW (eds). Pathology and Genetics of Tumours of Haematopoietic and Lymphoid Tissues. IARC Press: Lyon, France, 2001, pp 162-167.

17 Minowa $\mathrm{O}$, Arai T, Hirano $\mathrm{M}$, et al. Mmh/Ogg1 gene inactivation results in accumulation of 8-hydroxyguanine in mice. Proc Natl Acad Sci USA 2000;97: 4156-4161.

18 Mohamed AN, Palutke M, Eisenberg L, et al. Chromosomal analysis of 52 cases of follicular lymphoma with $\mathrm{t}(14 ; 18)$, including blastic/blastoid variant. Cancer Genet Cytogenet 2001;126:45-51.

19 Hoglund M, Sehn L, Connors JM, et al. Identification of cytogenetic subgroups and karyotypic pathways of clonal evolution in follicular lymphomas. Genes Chromosomes Cancer 2004;39:195-204.

20 Zuo C, Ai L, Ratliff $\mathrm{P}$, et al. $\mathrm{O}^{6}$-methylguanine-DNA methyltransferase gene: epigenetic silencing and prognostic value in head and neck squamous cell carcinoma. Cancer Epidemiol Biomarkers Prev 2004;13: 967-975.

21 Rossi D, Capello D, Gloghini A, et al. Aberrant promoter methylation of multiple genes throughout the clinico-pathologic spectrum of B-cell neoplasia. Haematologica 2004;89:154-164.

22 Rossi D, Gaidano G, Gloghini A, et al. Frequent aberrant promoter hypermethylation of $\mathrm{O}^{6}$-methylguanine-DNA methyltransferase and death-associated protein kinase genes in immunodeficiency-related lymphomas. Br J Haematol 2003;123:475-478.

23 Esteller M. Profiling aberrant DNA methylation in hematologic neoplasms: a view from the tip of the iceberg. Clin Immunol 2003;109:80-88.

24 Fan CY, Ai L, Zuo C, et al. DNA repair gene, hOGG1, in head and neck squamous cell carcinoma (HNSCC): gene expression and promoter methylation analysis in primary tumors and cancer cell lines. Mod Pathol 2004;17(Suppl):223A-224A.

25 Ashton-Key M, Diss TC, Isaacson PG, et al. A comparative study of the value of immunohistochemistry and the polymerase chain reaction in the diagnosis of follicular lymphoma. Histopathology 1995; 27:501-508

26 Nguyen PL, Zukerberg LR, Benedict WF, et al. Immunohistochemical detection of $\mathrm{p} 53$, bcl-2, and retinoblastoma proteins in follicular lymphoma. Am J Clin Pathol 1996;105:538-543.

27 Lai R, Arber DA, Chang KL, et al. Frequency of bcl-2 expression in non-Hodgkin's lymphoma: a study of 778 cases with comparison of marginal zone lymphoma and monocytoid B-cell hyperplasia. Mod Pathol 1998;11:864-869. 